\title{
Mediciones y análisis de vibraciones en el puente Virilla, ruta nacional $\mathrm{N}^{\circ} 1$
}

Measurements and analysis of vibrations at Virilla Bridge, national route $\mathrm{N}^{\circ} 1$

eBridge 2.0: sistema integrado para el desempeño de puentes

Francisco Navarro-Henríquez ${ }^{1}$, Daniel Ureña-Muñoz ${ }^{2}$

Fecha de recepción: 19 de agosto del 2014

Fecha de aprobación: 10 de octubre del 2014

Navarro-Henríquez, F; Ureña-Muñoz, D. Mediciones y análisis de vibraciones en el puente Virilla, ruta nacional $\mathrm{N}^{\circ} 1$. Tecnología en Marcha. Vol. 28, № 2, Abril-Junio. Pág 16-32.

1 Ingeniero en Electrónica, M.Sc., MBA, Profesor Investigador eBridge 2.0 sistema integrado para el desempeño de puentes. Instituto Tecnológico de Costa Rica. Teléfono: (506) 25509242. Correo electrónico: fnavarro@itcr.ac.cr

2 Ingeniero Civil con énfasis en estructuras, M.Sc., asesor en análisis de vibraciones eBridge 2.0 sistema integrado para el desempeño de puentes. Instituto Tecnológico de Costa Rica. Teléfono: (506) 8910-8072. Correo electrónico: durenamunoz@gmail.com 


\title{
Palabras clave
}

Dinámica estructural; análisis modal; Transformada Rápida de Fourier (FFT); sensores de fibra óptica FBG; sensores de esfuerzo y vibración.

\section{Resumen}

Este artículo presenta la experiencia generada durante la inspección detallada de la superestructura del puente Virilla, ruta nacional № 1, como parte del proyecto de investigación eBridge 2.0: sistema integrado para el desempeño de puentes y a solicitud del Consejo Nacional de Vialidad (CONAVI), con base en el Contrato interadministrativo entre el CONAVI y el Tecnológico de Costa Rica (TEC).

En este trabajo se realiza una descripción general del puente en estudio, así como una inspección inicial de la estructura y se dispone de un modelo analítico del puente para el análisis estructural.

Las mediciones realizadas permitieron cuantificar las magnitudes vibratorias y la deformación en varias secciones del puente, en condición de tránsito vehicular en servicio (comportamiento ambiental). Los resultados experimentales obtenidos se comparan con el modelado analítico computacional de la estructura y este a su vez con normas nacionales e internacionales.

\section{Keywords}

Structural Dynamics; modal analysis; Fast Fourier Transform (FFT); FBG fiber optics sensors; Strain and vibration sensors.

\begin{abstract}
This article presents the experience generated during the detailed inspection of the superstructure at the Virilla bridge, National Route $N^{\circ}$ 1, as part of the research project "eBridge 2.0: integrated bridge system performance", by request of the National Roads Authority (CONAVI) and based on the existing inter-contract between CONAVI and the Technological Institute of Costa Rica (TEC).

In this paper an overview of the bridge main features is described with an initial inspection of the structure is performed and also it is provided an analytical model of the bridge for structural analysis.

The measurements allowed quantifying the vibration magnitudes and deformation in various sections of the bridge, on condition of vehicular traffic service (environmental performance). The experimental results are compared with computational analytical modeling of the structure and also with national and international standards.

\section{Introducción}

El presente trabajo busca determinar algunas de las características dinámicas del puente sobre el río Virilla por medio de pruebas de vibración, para establecer una condición de referencia conocida de la estructura, previamente al proceso de rehabilitación al que se encuentra sometido.
\end{abstract}


Una vez concluida la obra de rehabilitación del puente, se contrastaría una nueva prueba de vibración con los datos de las mediciones de vibración originales anteriores al trabajo de restauración.

La prueba de vibración desarrollada permite la medición de las magnitudes de aceleración en las direcciones vertical, lateral y longitudinal, la determinación de las frecuencias naturales de la estructura, así como la deformación (esfuerzo dinámico) presente en algunos de los elementos de la superestructura. El esquema de instrumentación empleado define dos puntos de medición en la cercha central y otro punto de medición en una de las secciones laterales.

Los resultados obtenidos de la prueba experimental se comparan con el modelo analítico del puente, para verificar que el comportamiento del mismo se ajusta a las condiciones de diseño y a la normativa nacional e internacional.

Las pruebas de carga dinámicas no destructivas (NDT) permiten la medición de la respuesta de un puente sujeto a excitaciones predeterminadas sin causar cambios en la respuesta elástica de la estructura. Estas pruebas sirven para verificar el desempeño global del puente y el rendimiento de algunos de sus componentes (AASHTO, 2011).

En forma general, las pruebas dinámicas pueden clasificarse en tres grandes categorías, a saber: peso en movimiento (WIM), prueba de respuesta dinámica y prueba de vibraciones (AASHTO, 2011).

Una prueba de peso en movimiento abarca el conteo y pesaje de unidades e incluso ejes de tracción del tránsito vehicular regular. Luego, una prueba de respuesta dinámica busca, bajo tráfico normal o en condiciones controladas con un vehículo de prueba, determinar un estimado realista de la carga dinámica permitida y rangos de tensión de carga viva que pueden ser utilizados para la cuantificación de límites de carga y evaluación de fatiga. Las pruebas de vibración se utilizan para determinar características dinámicas del puente, tales como frecuencias naturales de vibración, forma modal y amortiguamiento.

En el caso de la labor desarrollada en el puente sobre el río Virilla, se puede indicar que corresponde a una prueba de vibración.

\section{Carácterísticas generales del puente}

El puente sobre el río Virilla, ubicado en la ruta nacional 1, en el distrito La Uruca, del cantón de San José en la provincia de San José, fue diseñado en 1960. Tiene una longitud de 160 metros, cuenta con dos secciones de 19,84 m cada una, su alineación es recta. Posee siete superestructuras (seis de vigas I de acero y una de cercha), dos bastiones y dos pilas. Inicialmente se diseñó para una carga HS 15-44 con las especificaciones AASHO 1957 (Ortiz et al., 2014).

En el año 2010 se diseñó el reforzamiento de la losa, utilizando las especificaciones AASHTO 2002 y una carga HS20-44 + 25\% (AASHTO, 2011).

La figura 1 muestra una fotografía con la vista del puente y en la figura 2 puede observarse un diagrama con la vista en planta y lateral del puente con los puntos de medición para vibración y esfuerzo de deformación. 


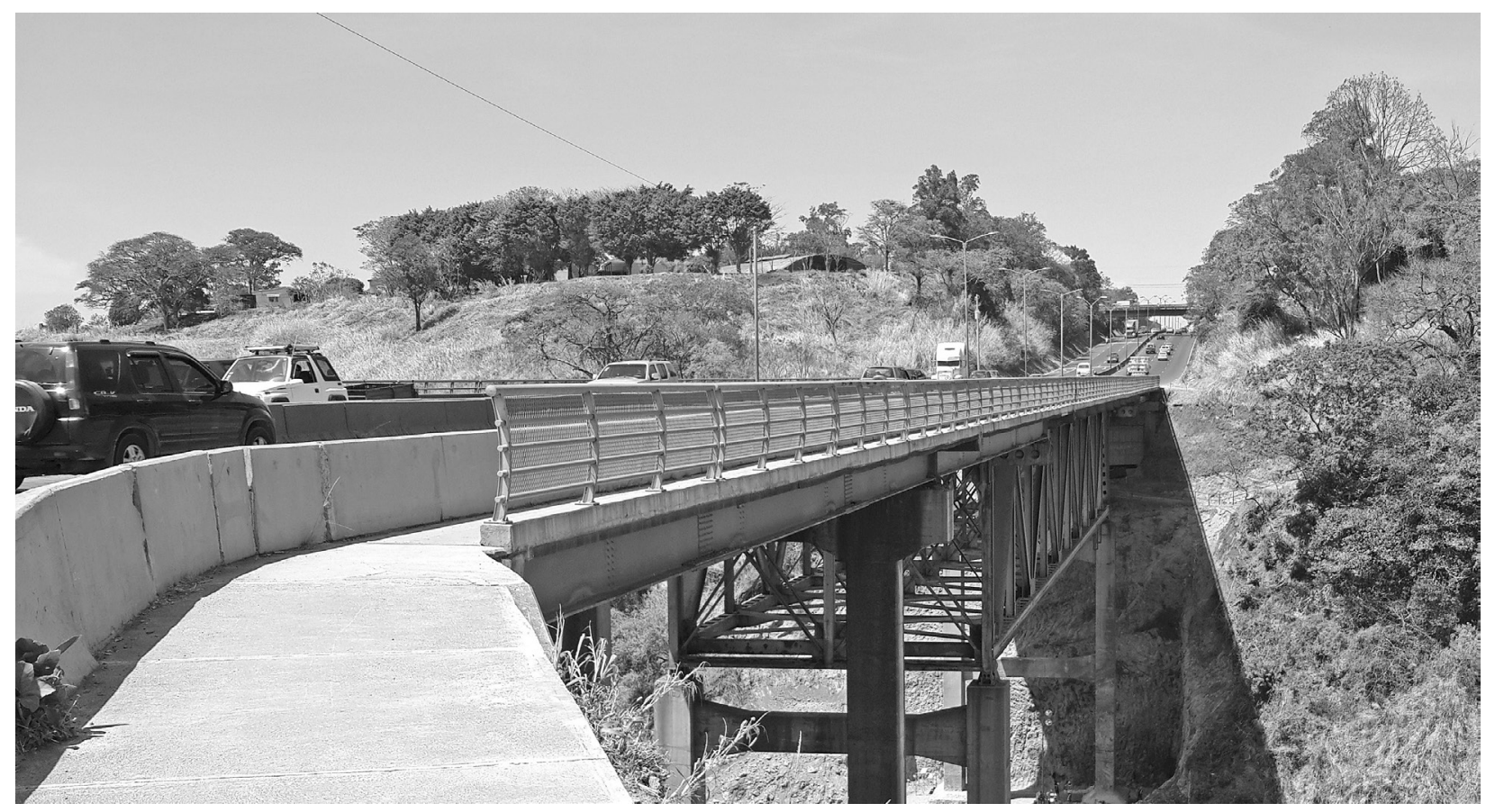

Figura 1. Vista del puente sobre el río Virilla, febrero 2014.

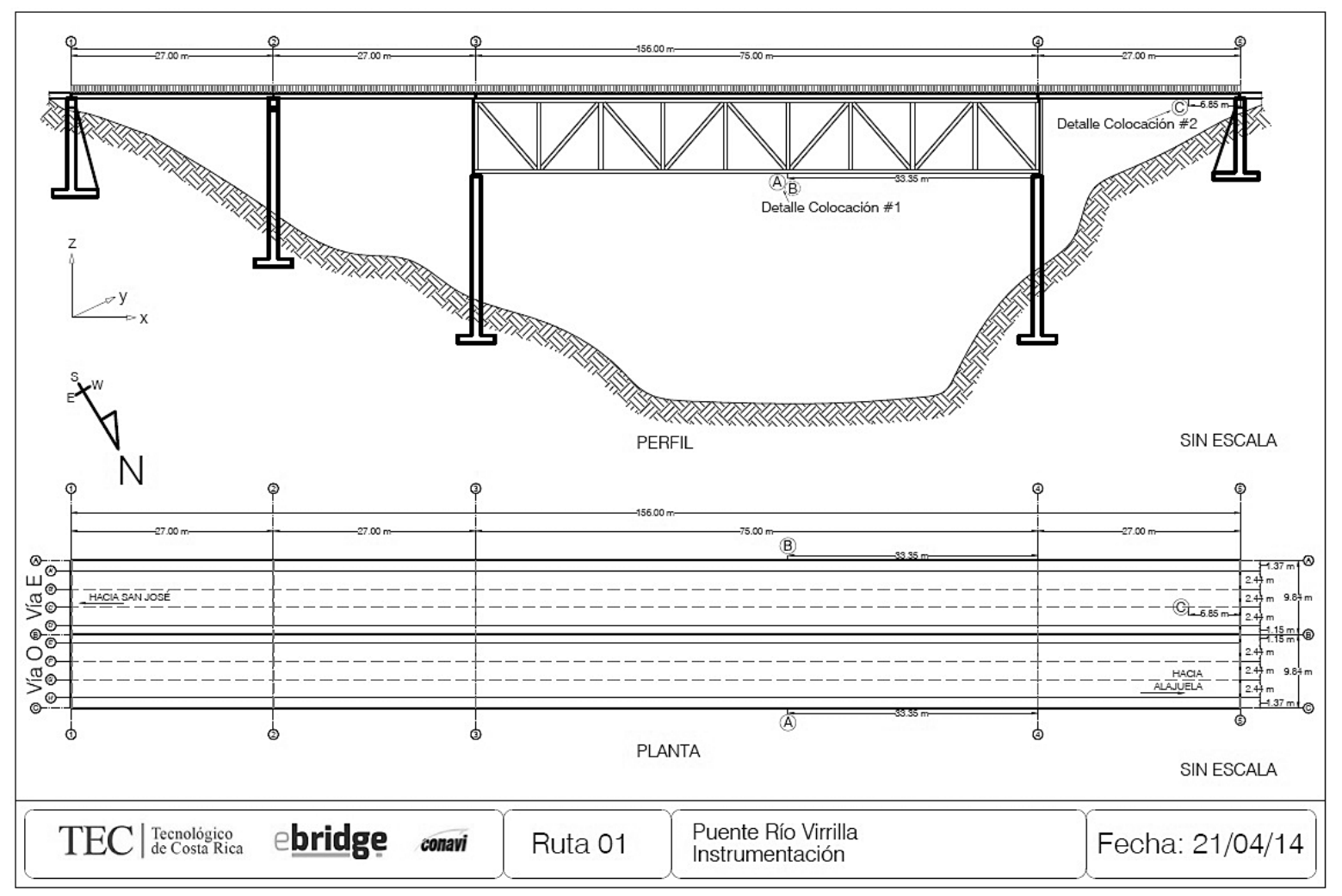

Figura 2. Vista en planta y pateral del puente sobre el río Virilla. Ubicación puntos de medición A, B y C. 


\section{Inspección inicial}

La estructura actualmente se encuentra en proceso de intervención para su reforzamiento, por esta razón el análisis se orientará únicamente hacia los elementos de acero de la superestructura. Las vigas principales en las seis superestructuras presentan problemas de oxidación, especialmente cerca de los apoyos, y la pintura se encuentra en mal estado. La vigas diafragmas en su mayoría presentan oxidación y alguna corrosión.

La cercha del puente muestra un mayor nivel de deterioro, encontrándose corrosión en muchos puntos y falta de mantenimiento básico, además presenta vibraciones y sonidos fuertes. Los apoyos tienen un alto nivel de deterioro.

El puente presenta un incremento significativo en la carga vehicular de diseño. El registro de tránsito promedio diario del año 2013 realizado por la Dirección de Planificación Sectorial de la Unidad de Estudios de Tráfico e Investigación del Ministerio de Obras Públicas y Transportes (MOPT) indica que el tránsito promedio diario (TPD) en el puente es de 87,407 vehículos.

El cuadro 1 presenta el desglose por tipo de vehículo y su participación respecto al total.

Cuadro 1. Distribución promedio diario del número de vehículos.

\begin{tabular}{|c|c|c|}
\hline Tipo de vehículo & \% del TPD & \# Vehículos diarios \\
\hline Particular & 75,22 & 65,748 \\
\hline Carga Liviana $^{*}$ & 11,02 & 9,632 \\
\hline Buses $^{*}$ & 4,43 & 3,872 \\
\hline 2 ejes & 5,73 & 5,008 \\
\hline 3 ejes $^{*}$ & 1,2 & 1049 \\
\hline 4 ejes $^{*}$ & 0 & 0 \\
\hline 5 ejes $^{*}$ & 2,4 & 2,098 \\
\hline TOTALES & 100 & 87,407 \\
\hline
\end{tabular}

El número promedio diario de camiones en un sentido por carril es de aproximadamente 3,100 unidades (Ortiz et al., 2014).

\section{Modelo analítico de puente}

Se construye un modelo estructural del puente con apoyo de la herramienta de software de análisis estructural SAP 2000.

Los planos consultados para la elaboración del modelo corresponden a los planos originales de construcción de la estructura, que representan la configuración con la cual ha funcionado el puente durante la mayor parte de su vida útil.

La figura 3 muestra la vista superior tridimensional del modelo estructural elaborado con SAP 2000. 


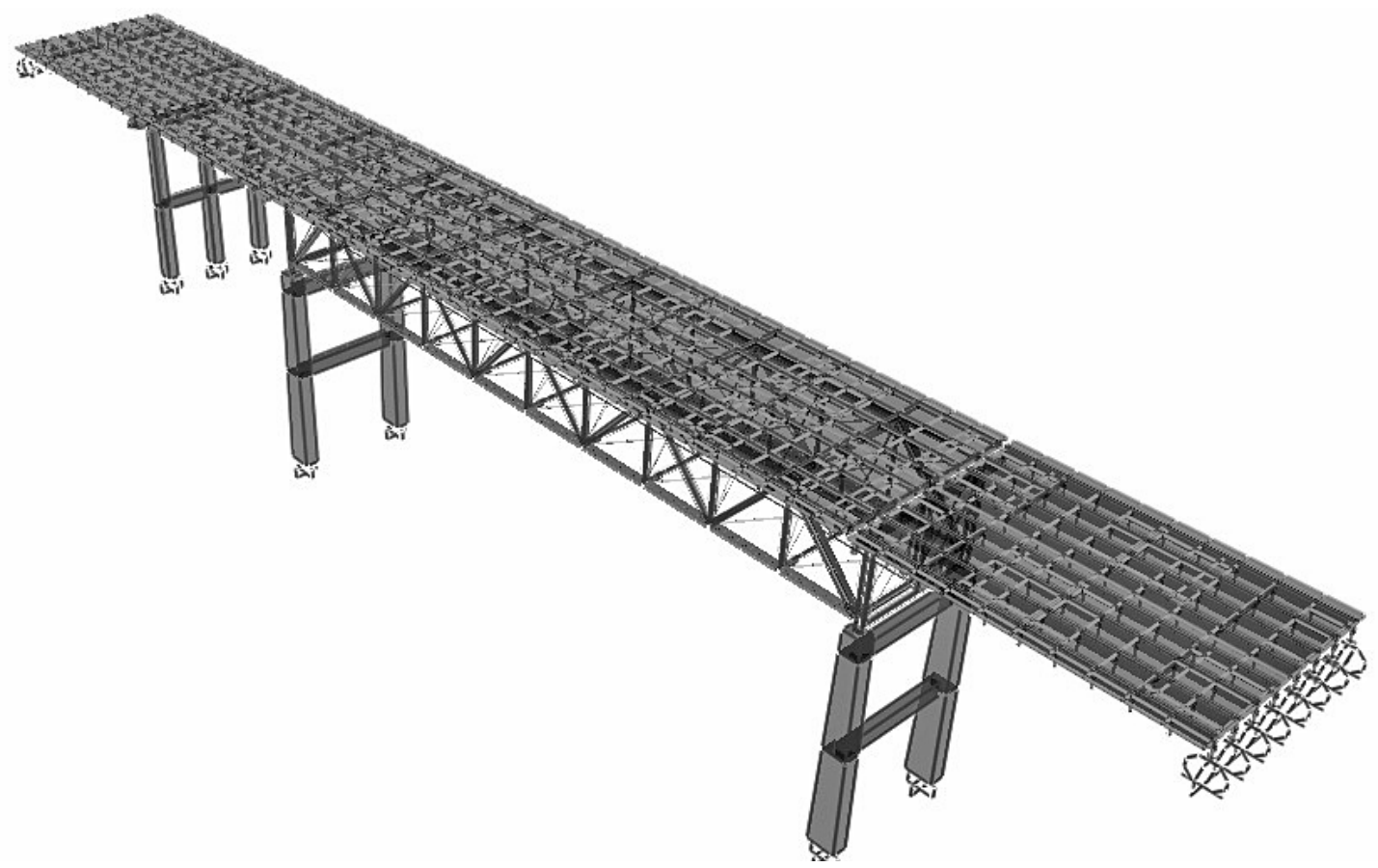

Figura 3. Vista superior tridimensional del modelo computacional.

La figura 4 muestra el modelo estructural del puente con la forma modal 9, cuya frecuencia natural de vibración teórica corresponde a $3.23 \mathrm{HZ}$. Esta frecuencia tiene participación en las direcciones $Y$ (transversal) y Z (vertical).



Figura 4. Modelo analítico. Modo de vibración y frecuencia.

\section{Estudio de vibraciones y esfuerzos dinámicos}

Las mediciones experimentales de vibración y esfuerzo dinámico se llevaron a cabo durante el mes de abril de 2014, permitiendo la circulación vehicular normal en servicio sobre el puente en ambas direcciones (comportamiento ambiental). 
Se establecieron tres puntos de medición en el puente, correspondientes a A, B y C, como se puede apreciar en la figura 2. En cada punto de medición se cuantificaron las magnitudes vibratorias y deformación (esfuerzo dinámico) por medio de la tecnología de sensores de fibra óptica FBG (Fiber Bragg Grating) (Kumar y Madhav, 2010).

Las mediciones de las aceleraciones se realizaron para cada punto en sentido longitudinal, transversal y vertical, que respectivamente corresponden a las coordenadas del modelo estructural, x, y, z. En todos los casos, el tiempo de medición fue de 30 minutos, capturando 1000 datos por segundo de cada sensor.

La instrumentación utilizada estuvo compuesta de un Interrogador óptico sm130 de 4 canales, $1 \mathrm{KHZ}$, un computador portátil con software para el registro de los datos ENLIGHT v1.5.59, un acelerómetro óptico de tes ejes como sensor de vibraciones, os7103, un medidor de deformación os3610 y un sensor de temperatura absoluta os4230 (Micron Optics, 2012).

En la figura 5 se muestra el esquema de instrumentación.

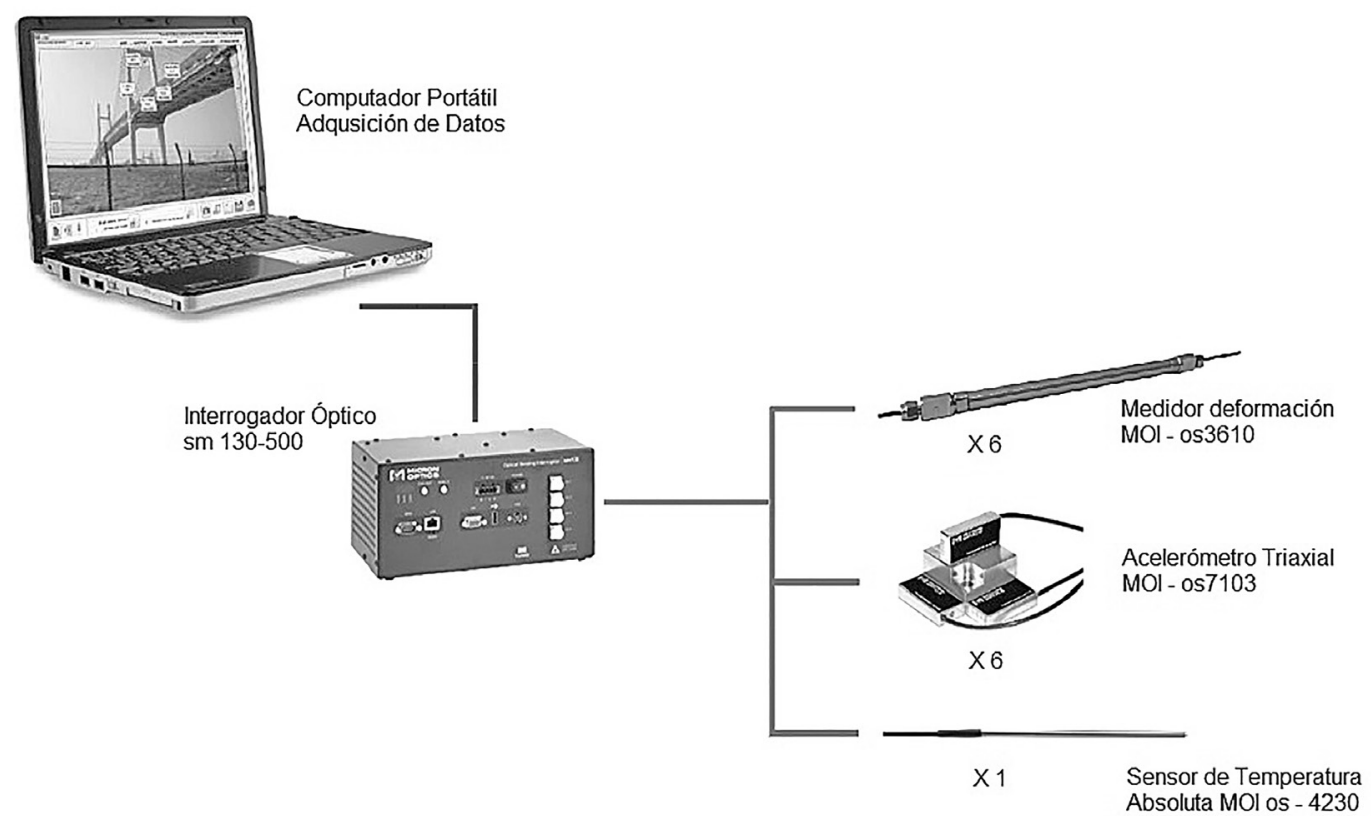

Figura 5. Esquema de instrumentación.

\section{Medidas de vibración}

Para cada una de las mediciones de vibración, dado que se tienen los valores de aceleraciones en función del tiempo, se procedió a aplicar filtros de paso de banda entre los $0.1 \mathrm{~Hz}$ a los $25 \mathrm{~Hz}$. Una vez aplicados dichos filtros con el fin de evitar vibraciones por ruido, se procedió a realizar la FFT (transformada rápida de Fourier) con el fin de determinar las frecuencias predominantes, las cuales se definen como frecuencias "in situ" (Davis et al., 1996).

La figura 6 muestra el registro de los datos de vibración y su correspondiente espectro de frecuencia en el punto de medición A. 
Vibración Eje X (Longitudinal) - Punto de medición A

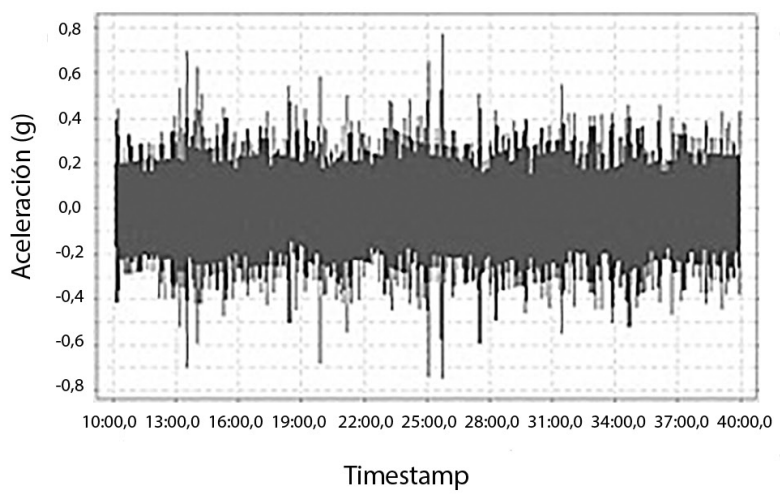

Vibración Eje Y (Transversal) - Punto de medición A
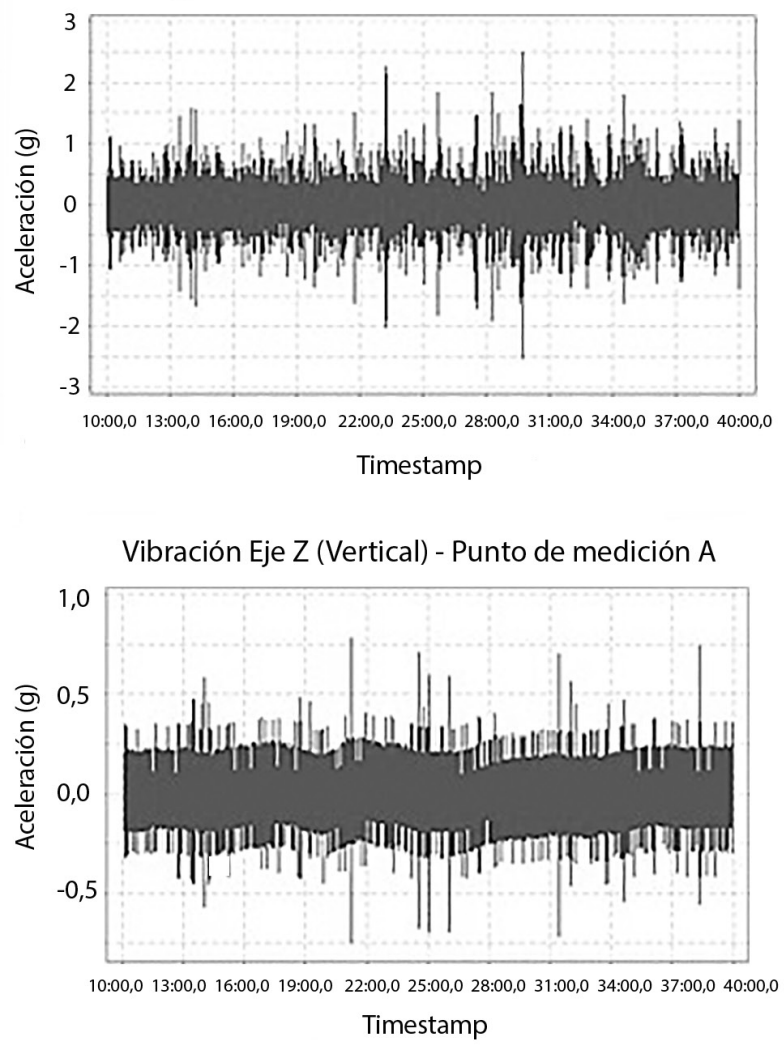
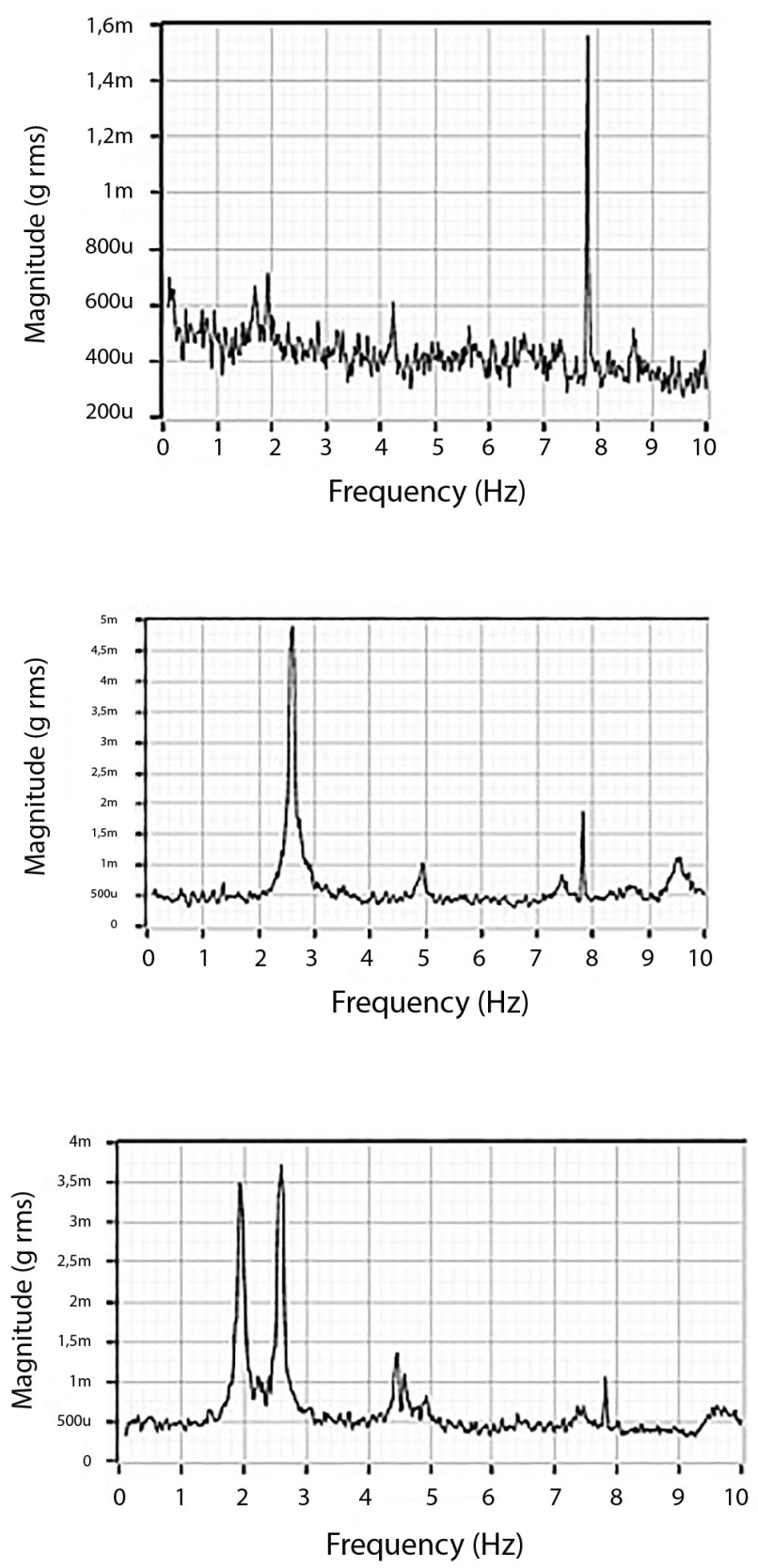

Figura 6. Señales de vibración y espectro de frecuencia, punto de medición A.

Una vez obtenidas las frecuencias "in situ", se procedió a compararlas con las frecuencias del modelo estructural computacional. Inicialmente se determinó la participación modal en cada una de las direcciones $\mathrm{x}, \mathrm{y}, \mathrm{z}$.

Del modelo analítico se obtuvieron los primeros 96 modos de vibración, sus participaciones en cada una de las tres direcciones y el porcentaje de participación con respecto al número de modo y a la frecuencia.

La figura 7 muestra una gráfica con la participación modal versus la frecuencia, obtenida por medio de la herramienta de software de análisis estructural SAP 2000. 


\section{Participación Modal}

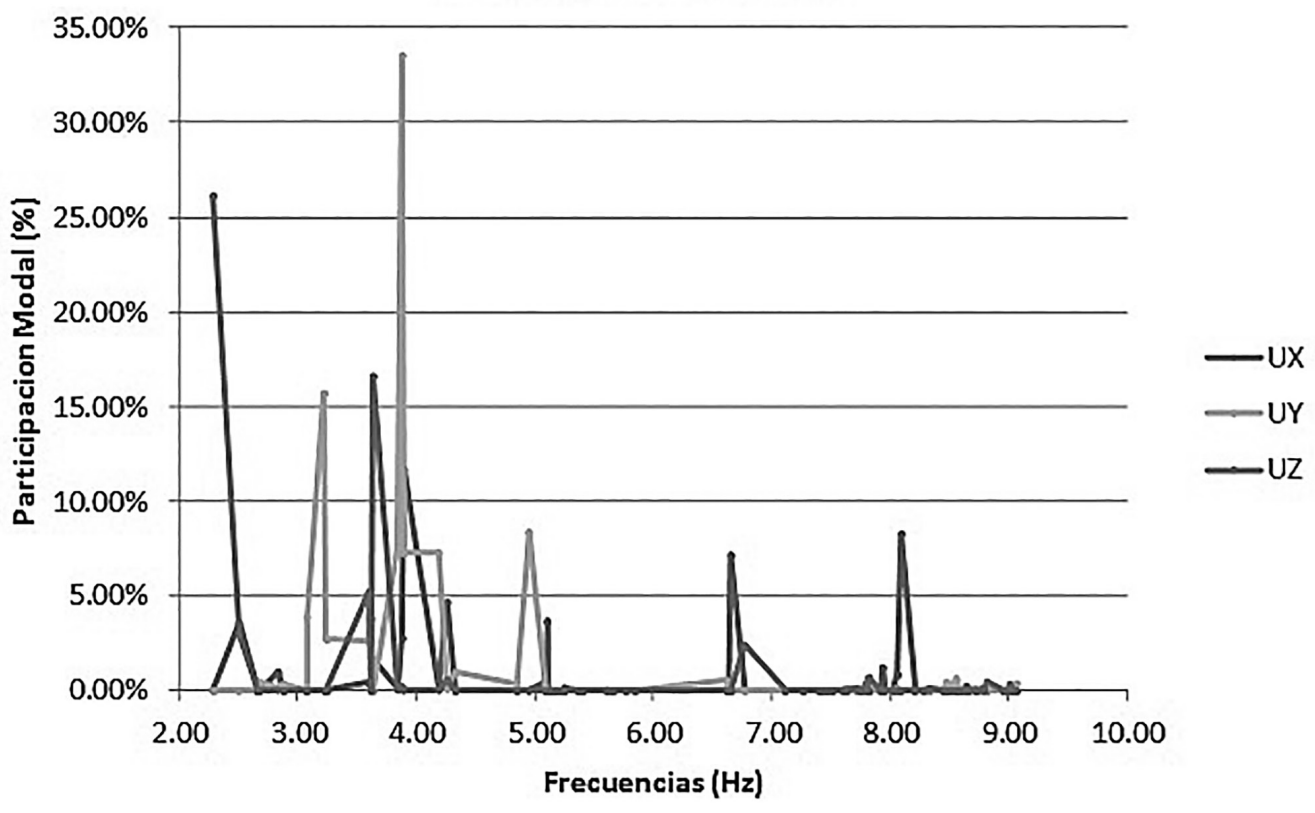

Figura 7. Participación modal vs frecuencia

Para cada uno de los puntos de medición en el puente se determinó su correspondiente nodo en el modelo. Luego, para cada uno de los nodos, se determinó el desplazamiento máximo modal, para cada uno de los modos, y posteriormente se comparó con su respectivo punto de medición experimental.

La figura 8 presenta la comparación de frecuencias experimentales con las del modelo estructural computacional en el punto de medición A correspondiente al nodo 7.

En el cuadro 2 y la figura 9 se presenta, para los puntos de medición A y B, los valores de las frecuencias identificadas experimentalmente y las del modelo estructural computacional. Los porcentajes de diferencia varían de un $4 \%$ a un $20 \%$. Dicha diferencia se debe a factores tales como que el modelo tiene una rigidez mayor a la que realmente tiene la estructura, los materiales se están consideran isotrópicos y con características mecánicas iguales, además los nodos pueden tener una rigidez diferente a la que se está considerando en el modelo; también podría deberse a respuesta no lineales en los materiales.

El cuadro 3 resume la estadística descriptiva en los puntos de medición para observar las magnitudes de los valores mínimos, máximos y rangos de las mediciones de vibración obtenidas.

Se determinaron las aceleraciones máximas en los lapsos de los gráficos en donde los picos máximos y mínimos son estables y no hay variaciones mayores a un 5\% (Wenzel, 2009). El cuadro 4 resume los resultados obtenidos. 

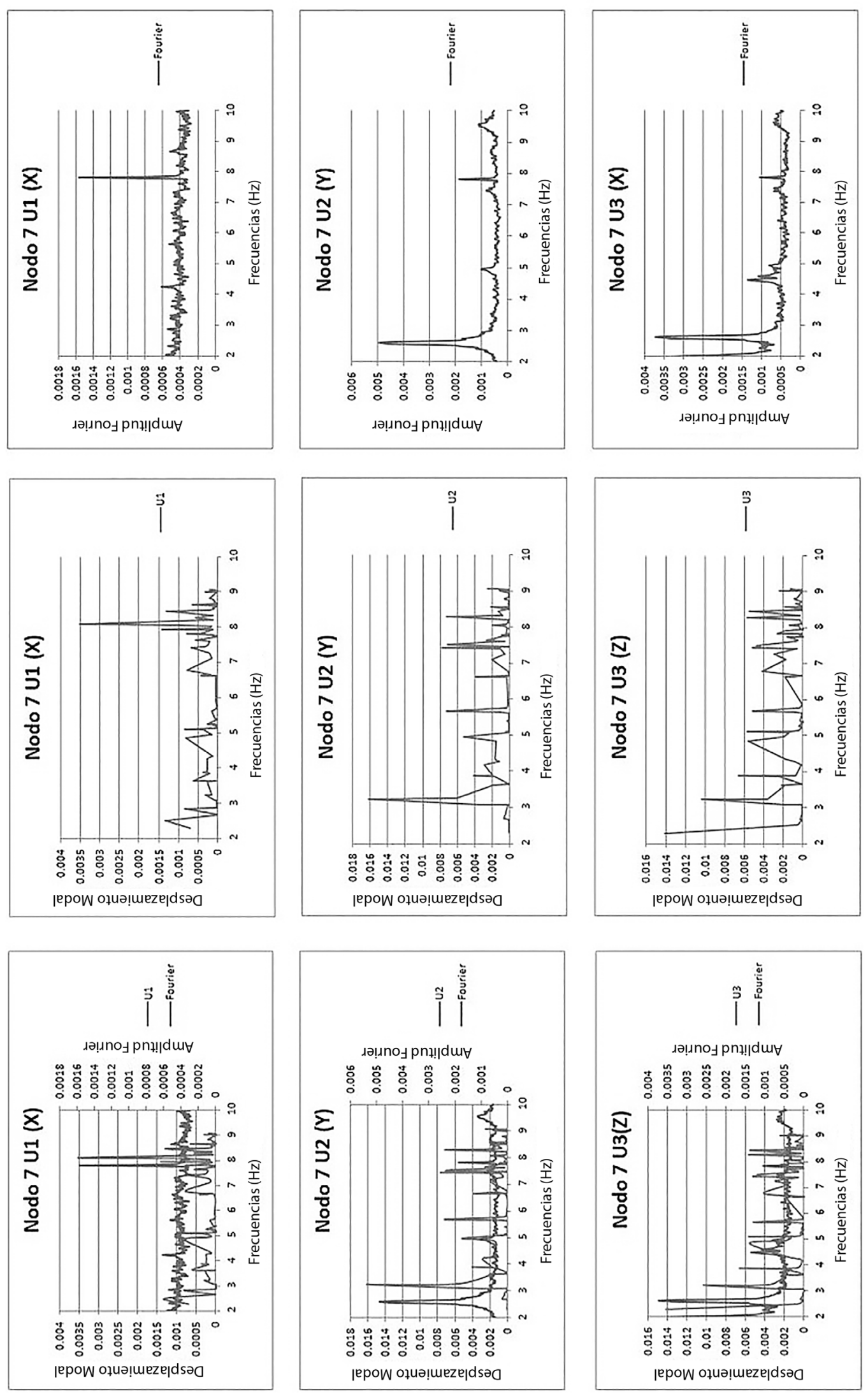

Figura 8. Comparación de frecuencias mediciones experimentales versus modelo analítico. Punto de medición A nodo 7. 
Cuadro 2. Frecuencias obtenidas para los nodos A y B

\begin{tabular}{|c|c|c|c|}
\hline Eje/Frec. & "in situ" & Mod. Est. & $\%$ Dif \\
\hline$X$ & 7.81 & 8.1 & $4 \%$ \\
\hline$Y, Z$ & 2.6 & 3.23 & $20 \%$ \\
\hline$Z$ & 1.96 & 2.23 & $12 \%$ \\
\hline
\end{tabular}

\section{Comparación frecuencias Nodos A y B}

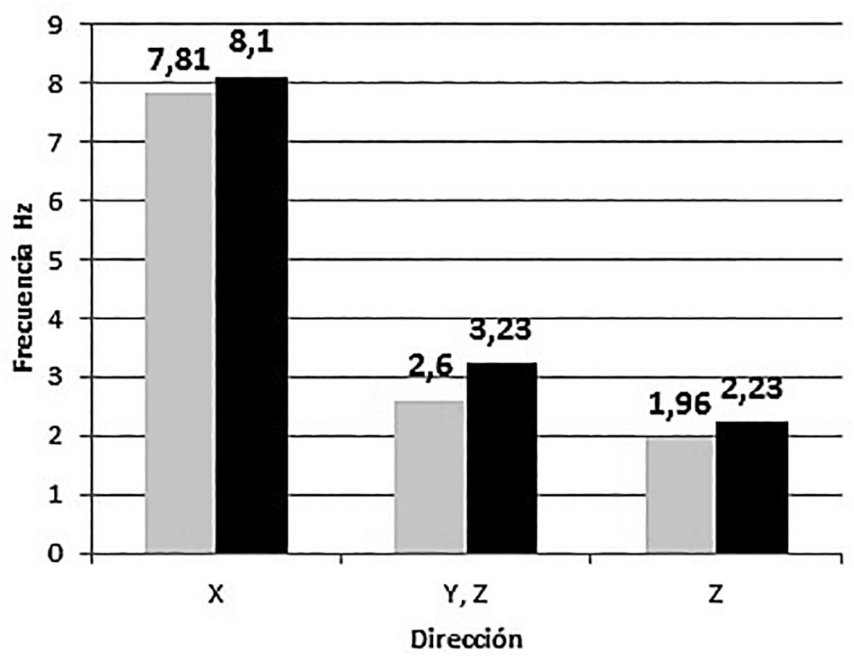

口"In Situ" घ Mod. Est.

Figura 9. Comparación de frecuencias nodos A y B

Cuadro 3: Resumen de mediciones de vibración en puntos A, B y C

\begin{tabular}{|c|c|c|c|c|}
\hline Pto. medición & Vibración & Mínimo & Máximo & Rango \\
\hline \multirow{3}{*}{$A$} & eje $X$ & $-0,747$ & 0,767 & 1,514 \\
\cline { 2 - 5 } & eje $Y$ & $-2,52$ & 2,512 & 5,032 \\
\cline { 2 - 5 } & eje Z & $-0,752$ & 0,783 & 1,535 \\
\hline \multirow{2}{*}{ B } & eje $X$ & $-0,847$ & 0,769 & 1,616 \\
\cline { 2 - 5 } & eje $Y$ & $-2,195$ & 2,044 & 4,239 \\
\cline { 2 - 5 } & eje Z & $-0,959$ & 0,889 & 1,848 \\
\hline \multirow{2}{*}{ C } & eje $X$ & $-9,496$ & 9,621 & 19,117 \\
\cline { 2 - 5 } & eje $Y$ & $-10,002$ & 9,996 & 19,998 \\
\cline { 2 - 5 } & eje Z & $-5,691$ & 7,208 & 12,899 \\
\hline
\end{tabular}


Cuadro 4: Aceleraciones máximas y mínimas en tramo cercha

\begin{tabular}{|c|c|c|c|}
\hline \multirow{2}{*}{ Eje } & Aceleración & Nodo A & Nodo B \\
\hline \multirow{2}{*}{ Vertical (z) } & Máxima & $0.27 \mathrm{~g}$ & $0.33 \mathrm{~g}$ \\
\cline { 2 - 4 } & Mínima & $0.30 \mathrm{~g}$ & $0.35 \mathrm{~g}$ \\
\hline \multirow{2}{*}{ Longitudinal (x) } & Máxima & $0.37 \mathrm{~g}$ & $0.39 \mathrm{~g}$ \\
\cline { 2 - 4 } & Mínima & $0.38 \mathrm{~g}$ & $0.37 \mathrm{~g}$ \\
\hline \multirow{2}{*}{ Transversal (y) } & Máxima & $0.55 \mathrm{~g}$ & $0.57 \mathrm{~g}$ \\
\cline { 2 - 4 } & Mínima & $0.56 \mathrm{~g}$ & $0.62 \mathrm{~g}$ \\
\hline
\end{tabular}

Los datos de las frecuencias de vibración medidas en sitio y la amplitud de las aceleraciones se introducen en gráficos de referencia que contienen diversos criterios de aceptación. La figura 10 muestra el caso de confort, en el cual para una aceleración máxima vertical de 0,35 g, con una frecuencia natural de vibración de $1,96 \mathrm{~Hz}$ (punto rojo), se ubica en la zona de intolerable a partir de 5-20 minutos (Wenzel, 2009).

La figura 11 presenta otro criterio de revisión, correspondiente al presentado por Chang (1973), en el que para la misma aceleración máxima vertical 0,35 g, con la frecuencias natural de vibración de 1,96 Hz, se tiene un nivel de entre D2 y D3, lo cual está entre ligeramente incómodo y extremadamente incómodo. Evidentemente es perceptible y la situación se corroboró en sitio.

La figura 12 es una propuesta tentativa como resumen general de criterios que proporciona un indicador crudo pero simple que, por supuesto, no se puede aplicar a ciegas a todas las estructuras. En términos generales, los criterios de aceptación de confort son mucho más estrictos que los criterios estructurales y por lo tanto se vuelven más críticos para fines de diseño estructural (Wenzel, 2009).

En el punto de medición A, con una aceleración pico vertical de 0,783 g y una frecuencia natural de 1,96 Hz (punto rojo), el valor pico de la vibración registrada en el eje z se localiza en una zona de percepción pero lejos del criterio estructural. 


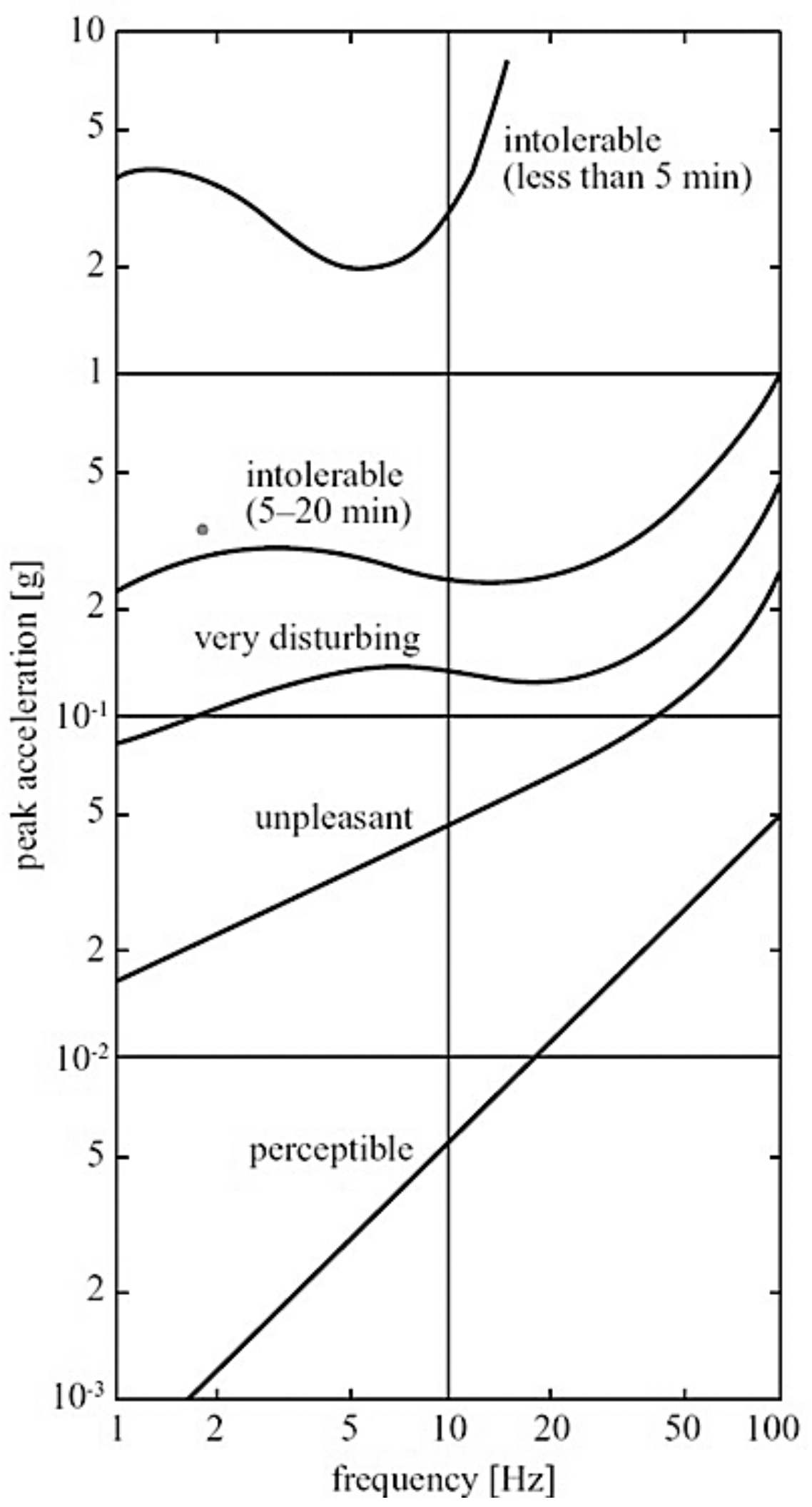

Figura 10. Tolerancias Confort para aceleraciones pico 


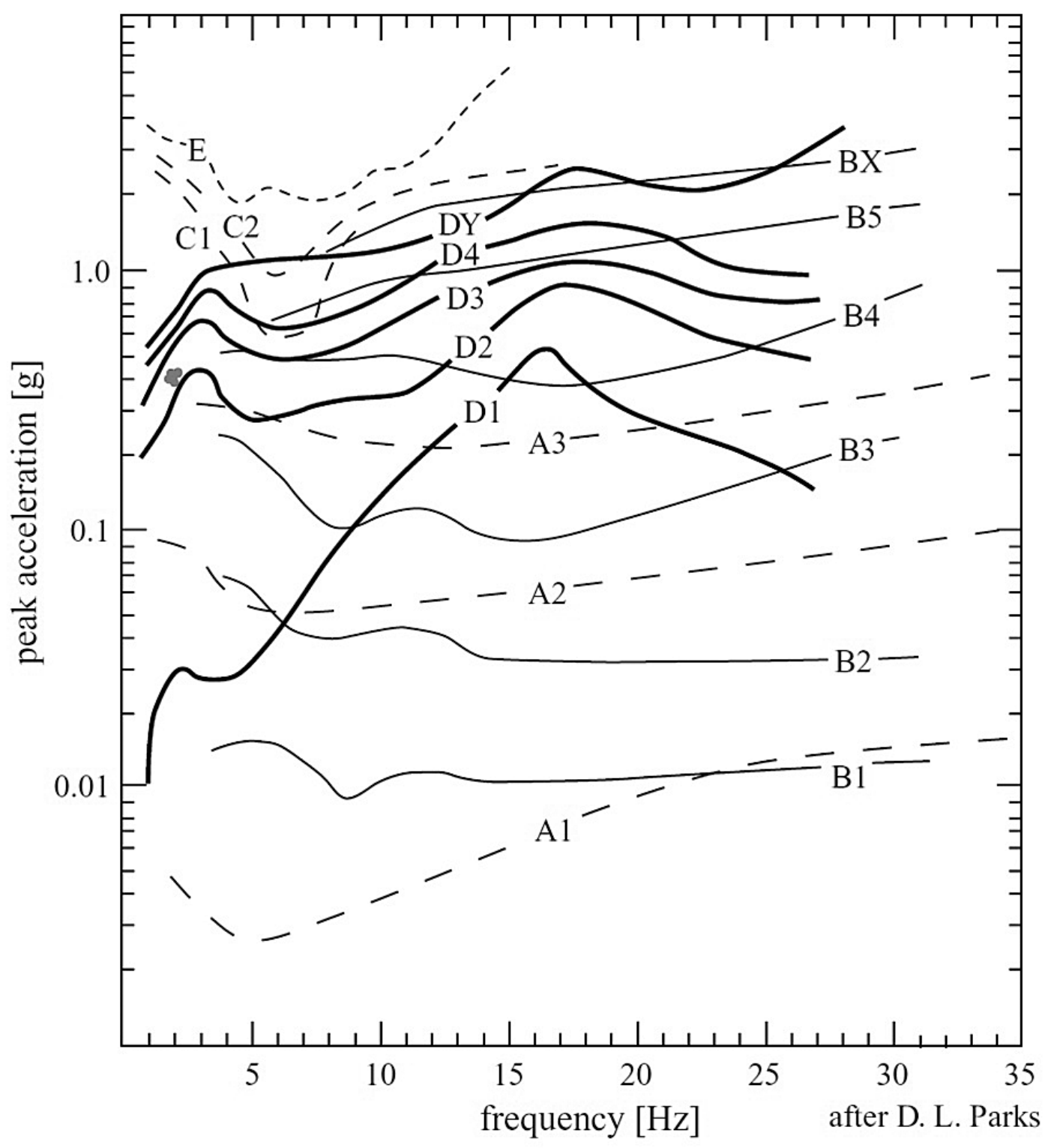
A Goldman (1948)
A1 Perceptible
C Magid \& Coeman (1960)
A2 Unpleasant
C1 3 min tolerance limit
A3 Intolerable
C2 1 min tolerance limit
B Gorill \& Snyder
B1 Treshold of perception
D Parks (1961)
B2 Definitely or easily perceptible
B3 Irritating or annoying
D1 Definitely perceptible
D2 Mildly annoying
D3 Extremely annoying
D4 Alarming
B4 Max tolerable for cont. operation
B5 Intolerable
BX Highest intensity endured
DY Highest intensity called alarming
E Zeigenruecker \& Magid (1959)
Short time tolerance

Figura 11. Estudio de percepción de las aceleraciones (Chang 1973) 


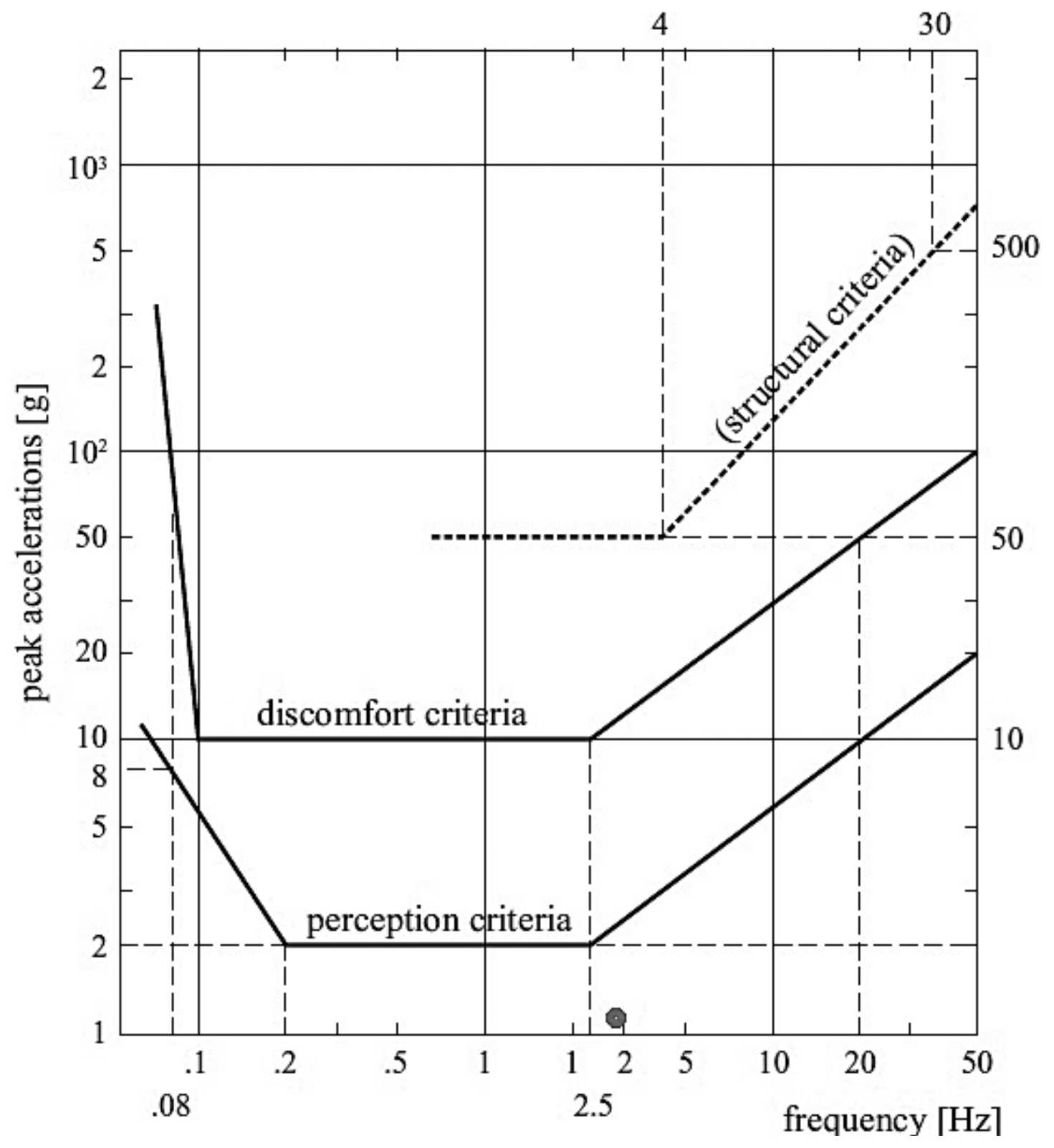

Figura 12. Criterio de aceleración pico

\section{Medidas de esfuerzos dinámicos}

La figura 13 muestra el registro de los datos de deformación dinámica en el punto de medición A. Se obtiene para este caso una deformación media de 1,27 ue con un valor mínimo de -11,12 ue y un valor máximo de 38,15 $\mu \mathrm{e}$. El valor de percentil 95 para el esfuerzo de tensión dinámica es de 7,6 $\mathrm{\mu e}$ (microstrain).

El cuadro 5 muestra los esfuerzos máximos calculados según las mediciones de deformación unitaria dada por los sensores, y los esfuerzos máximos en los mismos puntos provenientes del modelo teórico para fatiga. 


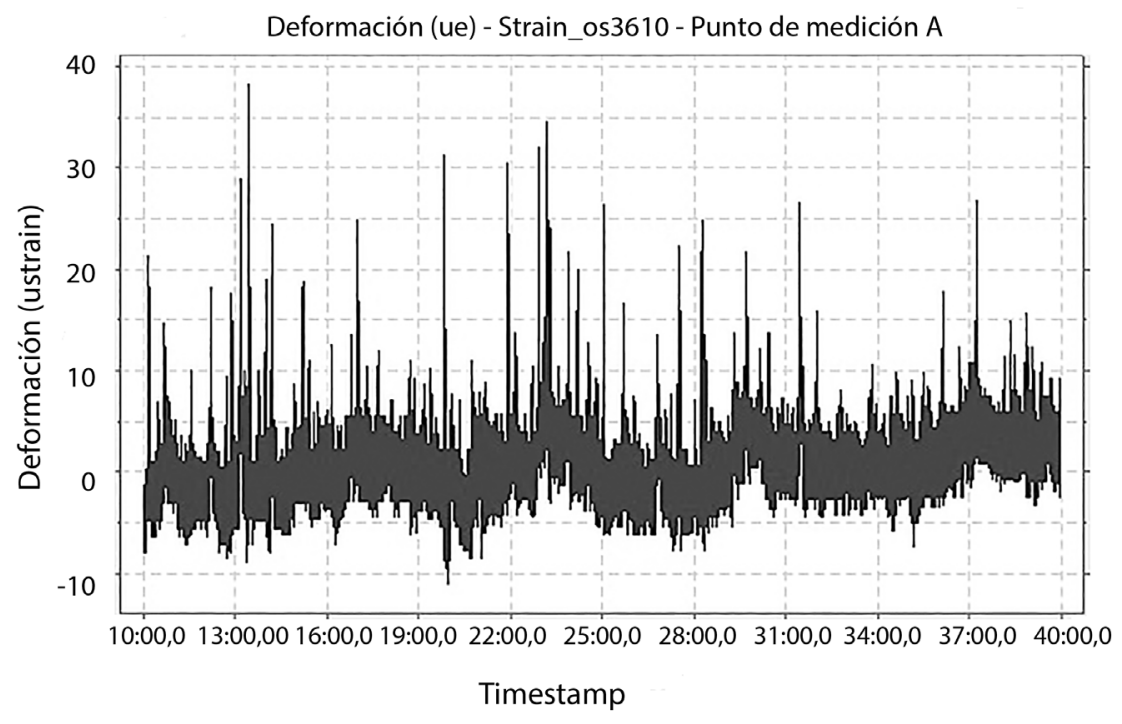

Figura 13. Deformación dinámica el punto de medición A

Cuadro 5. Esfuerzos máximos en los puntos A, B y C

\begin{tabular}{|c|c|c|c|}
\hline \multirow{2}{*}{ PUNTO } & $\varepsilon(\mu \varepsilon)$ & $\sigma$ & $\sigma_{\text {Modelo }}$ \\
\cline { 2 - 4 } & Percentil 95\% & $\mathrm{kg} / \mathrm{cm}^{2}$ & $\mathrm{~kg} / \mathrm{cm}^{2}$ \\
\hline A & 7,6 & 15,96 & 85,4 \\
\hline B & 104,27 & 218,97 & 89,3 \\
\hline C & 128,4 & 269,64 & 197,1 \\
\hline E $=$ & $2.10 \mathrm{E}+06$ & $\mathrm{~kg} / \mathrm{cm}^{2}$ & \\
\hline
\end{tabular}

Aunque los esfuerzos mostrados en el cuadro se obtuvieron para cargas distintas, ya que en el modelo teórico la carga es la de un vehículo de prueba de diseño y la deducida de los datos es una carga no cuantificada, se tiene que el orden de magnitud es similar y ratifica la validez del modelo teórico.

\section{Conclusiones}

- A partir de la excitación dinámica del tránsito regular de vehículos en el puente, fue posible obtener frecuencias naturales de vibración. Esto representa una ventaja al no ser necesario suspender este tráfico para realizar pruebas de impacto o de un excitador dinámico con frecuencia conocida para obtener la respuesta dinámica.

- De las mediciones de frecuencias en sitio se determinó que ante excitaciones dinámicas debido al tránsito regular de vehículos, el puente experimenta aceleraciones altas simultáneas en las tres direcciones (situación que se observó en sitio), lo cual no es normal en este tipo de estructuras. 
- Para el tramo de cercha, en los nodos A y B, se determinaron las frecuencias de 7,8 Hz en la dirección longitudinal " $x$ ", 2,6 Hz en las direcciones transversal y vertical "y" y " $z$ ", y de $1,96 \mathrm{~Hz}$ en la dirección vertical "z". Para el tramo de vigas, en el nodo C, se determinaron las frecuencias de $7,8 \mathrm{~Hz}$ en la dirección longitudinal " $x$ ", 2,95 Hz en las direcciones transversal "y", y de 2,65 Hz, 2,95 Hz y 8,36 Hz en la dirección vertical "z".

- El comportamiento deducido del modelo de análisis teórico es similar y comparable al obtenido de los datos recopilados en sitio. Así, las frecuencias obtenidas en sitio se validan con el modelo estructural, las diferencias porcentuales son aceptables ya que en el modelo estructural no se puede reflejar en su totalidad el estado de rigidez actual del puente. Dichas diferencias se deben a factores como que el modelo tiene una rigidez mayor a la que realmente tiene la estructura, los materiales se están considerando isotrópicos y con características mecánicas iguales, además los nodos pueden tener una rigidez diferente a la que se está considerando en el modelo, también podría deberse a respuestas no lineales en los materiales.

- En el caso de los nodos A y B, se obtiene que su comportamiento evaluado en forma independiente es similar entre sí, ya que las frecuencias obtenidas en las tres direcciones son prácticamente iguales.

- Para las aceleraciones máximas determinadas para el tramo de cercha, al revisarlo con los criterios de aceptación, se obtiene que numéricamente se demuestra que es perceptible (situación que se confirmó en sitio), que está fuera de los niveles de confort, que presenta niveles que van de ligeramente a extremadamente molesto, que es intolerable a partir de 5 a 20 minutos, y que si no se le presta atención a dicha vibración podría llegar a estar en un nivel alarmante o cabe la posibilidad a futuro de que aparezcan algunos daños o fisuras debido a la intensidad de la vibración inducida por las cargas vivas, en las zonas de máximos esfuerzos en tensión de la superestructura.

- Resulta relevante darle seguimiento al comportamiento dinámico de la estructura en forma periódica anual, realizando las mediciones de vibración en los mismos puntos, con la finalidad de prevenir desviaciones importantes de las frecuencias naturales de vibración que evidencien cambios o algún tipo de daño en la estructura.

\section{Bibliografía}

AASHTO (American Association of State Highway and Transportation Officials). (2011). Non-destructive load testing (pp. 8.1-8.16). En The Manual for Bridge evaluation. 2 ed. Washington, D.C.: AASHTO Publications office.

Chang, F. (1973). Human response to motions in tall buildings. Proceedings of the American Society of Civil Engineers, 98, 1259-1272.

Davis, M., Bellemore, D., Berkoff, A., Kersey, A., Putman, M., Idriss, R., Kodinduma, M. (1996). Fiber optic sensor system for bridge monitoring with both static load and dynamic modal sensing capabilities. Recopilado el 2 de septiembre de 2013 de la base de datos SPIE Digital Library de la sociedad internacional de óptica y fotónica. Disponible en http://spiedigitallibrary.org

Kumar, K. \& Madhav, V. (2010). Review on developments in fiber optical sensors and applications. Recopilado el 20 de agosto de 2013 de la base de datos SPIE Digital Library de la sociedad internacional de óptica y fotónica. Disponible en: http://spiedigitallibrary.org

Micron Optics, Inc. (2012). Products Sensing Solutions Sensors. Obtenido de http://www.micronoptics.com/products/ sensing_solutions/sensors/

Ortíz, G., Carranza, M., Navarro, F., Ureña, D., Vargas, K. (2014). Informe inspección detallada sobre superestructura para identificar evidencia de fatiga, Puente sobre Río Virilla, ruta nacional No. 1. Cartago: Instituto Tecnológico de Costa Rica.

Wenzel, H. (2009). Health Monitoring of Bridges. Viena: John Wiley \& Sons, Ltd 